\title{
SLEEP DISORDERS IN CHILDREN
}

\author{
Tony Jaffa, Stephen Scott, Jean Harris Hendriks, Colin M Shapiro
}

\section{Development of normal sleep}

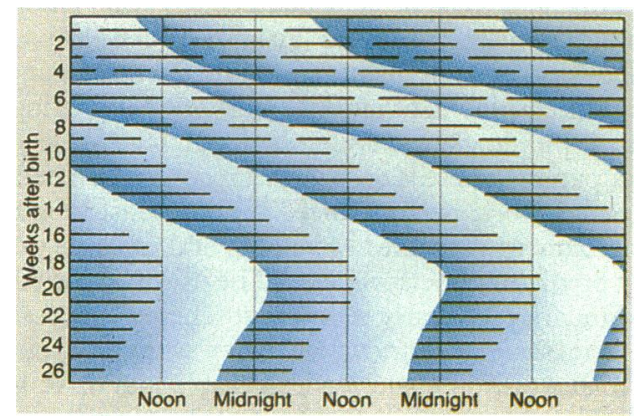

Initially an infant has a broken sleep regimen. After the first 12 weeks of life this usually consolidates into a regular sleep pattern.

Sleep is influenced by many factors, including age. Newborn babies sleep for about 16 hours a day with brief alternating periods of sleep and wakefulness, the electroencephalographic trace going directly from the alert pattern to rapid eye movement (REM) activity, missing out sleep stages 1-4. As babies become older they sleep for longer periods, though overall for fewer hours, and the sleep becomes concentrated into the night. By 3 months of age about $70 \%$ of babies sleep through until morning, though at 1 year $10 \%$ are still waking their parents each night. Sleep studies have shown that waking during the night is a fairly usual pattern, but what is more variable is whether the infant goes back to sleep or becomes more alert and calls out. By the age of 1 year the sleep electroencephalogram resembles that of an adult.

\section{Sudden infant death syndrome}

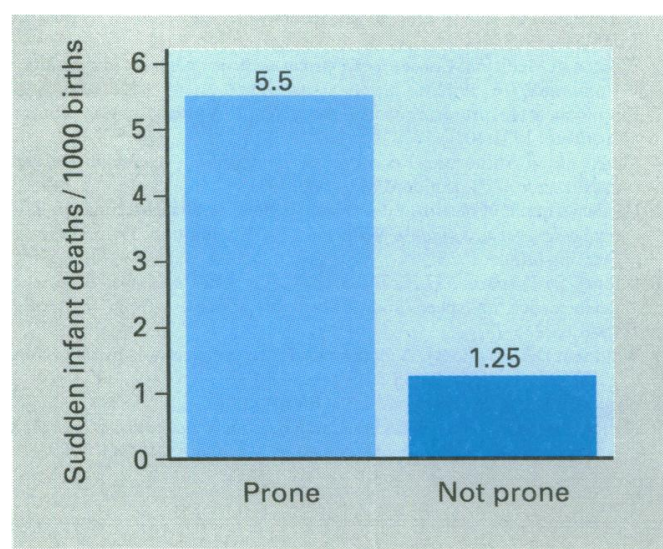

Effect of sleeping position on death rate from sudden infant death syndrome: results of a large prospective study in Tasmania.
Many physiological changes occur during sleep. These include variations in the regulation of breathing, circulation, and other autonomic functions. For example, thermoregulation is suspended during REM sleep, when cerebral blood flow increases dramatically. How any of these is precisely related to the sudden infant death syndrome is so far not known, though there are many theories. The deaths usually occur during sleep; over four fifths occur before the age of 6 months, and the rate is double between January and March compared with June to August. Retrospective studies both in the United Kingdom and abroad have shown significantly fewer deaths among babies who did not sleep prone. The effect of a campaign during which parents were told to place their babies on their backs or sides to sleep led to a remarkable reduction in the number of deaths in Avon, England. Late in 1991 this became a national policy in England and Wales, formally sponsored by the Department of Health, and results so far suggest a reduction nationally of about a half compared with 1988 .

Sleep is also a time of growth, which is facilitated by the establishment of early circadian rhythms. In young children who have been severely abused there is inhibition of nocturnal release of growth hormone, and psychosocial dwarfism may be the consequence. During the growth spurt in adolescence the steady decline in hours slept each night compared with childhood is temporarily reversed, often giving rise to misplaced complaints of lazy adolescents who do in fact require more sleep. 


\section{Patterns of sleep disturbance}

\section{Patterns of sleep disturbance}

\section{Excessive sleep:}

Sleep apnoea with daytime drowsiness

Klein-Levin syndrome

Narcolepsy

\section{Interrupted sleep:}

Nightmares

Night terrors

Sleepwalking and sleeptalking

Problems in settling and remaining asleep



Characteristic sleeping position of a child with sleep apnoea.

\section{Causes of sleep problems in children}

\section{The child}

Physical factors - Bedwetting; physical illness; developmental delay or handicap

Psychological factors - Established habitual cycle of night waking; anxiety about separation from parents; fears and worries; psychiatric disturbance-for example, depression, drug abuse; temperament

\section{The parents}

Failure to establish a bedtime routine

Rewarding response to child's waking

Emotional overinvolvement with the child

\section{The environment}

Cold, damp, or dark bedroom

Noise

Overcrowding

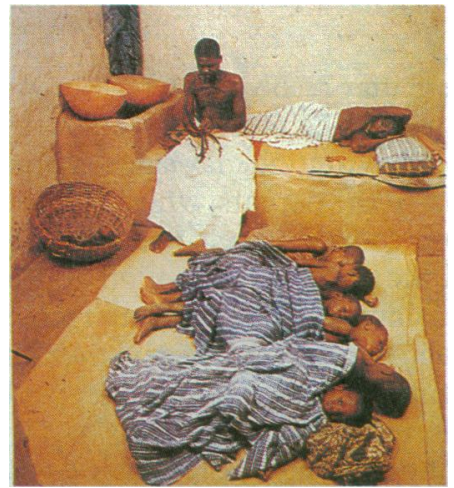

Living conditions may influence sleep.
Sleep disturbance can be broadly categorised into excessive sleep and inadequate or interrupted sleep.

\section{Excessive sleep}

Daytime drowsiness is most commonly the result of sleep deprivation, but it may also be caused by a number of rare syndromes that usually present from late childhood onwards.

Narcolepsy-This is characterised by a sudden irresistible desire to go to sleep that lasts for a few minutes at a time. It is associated with cataplexy (the sudden loss of tone in one or more muscle groups) and with vivid auditory or visual hallucinations when falling asleep. These can be extremely disturbing, and a child who complains of odd experiences at night should not be dismissed as merely dreaming.

Klein-Levin syndrome-This affects adolescent boys. Periods of pronounced hypersomnia and overeating alternate with periods of normality.

Sleep apnoea-Excessive tonsillar and adenoidal tissue may cause intermittent obstruction of the airway, which leads to sleep apnoea, daytime drowsiness, and even intellectual impairment. Children characteristically sleep on their elbows and knees with their buttocks in the air and neck hyperextended. Management is surgical, after which a growth spurt may be noted.

\section{Inadequate or interrupted sleep}

Nightmares occur during REM sleep. The child wakes in an anxious state with a tachycardia and tachypnoea and can usually remember at least some of the content of the dream. This may be related to specific traumatic experiences or to frightening films or television programmes. Nightmares are common, but unless they are frequent and persistent reassurance and comfort are all that is required.

Night terrors occur in about $3 \%$ of children, more commonly in boys, and tend to run in families. They occur in sleep stages 3 and 4-that is, in deep sleep. A child who experiences a night terror seems terrified, stares into space, and possibly mutters indistinctly. The child is not awake and if left alone will calm down and return to a resting sleep state. Though parents find a night terror disturbing, the child wakes in the morning with no memory of it. Night terrors increase at times of stress, but in isolation are not indicative of emotional disturbance; most children grow out of them.

Sleepwalking and sleeptalking, like night terrors, occur during sleep stages 3 and 4, are not remembered in the morning, and are not indicative of emotional disturbance. Parents of a habitual sleepwalker are well advised to secure doors and windows to reduce the risk of injury.

\section{Problems of settling and remaining asleep}

Much commoner than the specific conditions described above are general difficulties in settling and remaining asleep. The problem may be in settling, with long and increasingly acrimonious arguments about going to bed, after which children may repeatedly come out of their bedrooms. The problem may be later, when they wake up in the night and complain that they are afraid, or in pain, and it takes considerable reassurance before they go back to bed or get into bed with the parents. There are many contributory causes:

Physical and environmental factors must be considered. Children may be woken by somatic symptoms such as abdominal pain, the cough or breathlessness of asthma, or the irritation of eczema. Wetting the bed or worry that they are going to may cause considerable loss of sleep for enuretic children. There is much temporal variation in how much children sleep, and those at the extreme who wake frequently as babies tend to continue to sleep less than other children as they grow older. Sleep disturbance is relatively common among those with appreciable developmental delay and mental handicap.

Unfavourable living conditions associated with social deprivation such as a cold, damp bedroom, or sharing the room or bed with noisy siblings, will exacerbate if not cause sleep disturbances. 


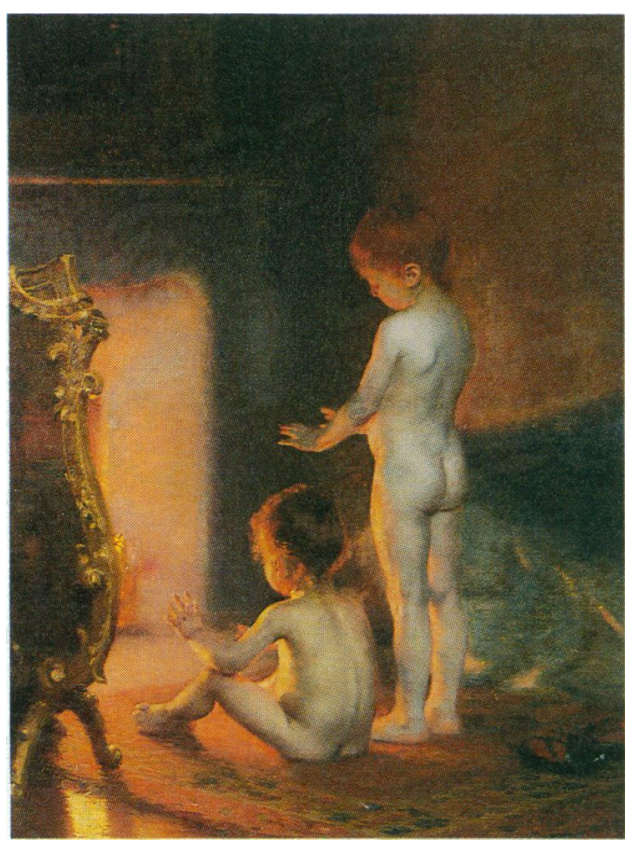

Comforting routines before bedtime help sleep. ("After the bath" by Paul Peel (1890)).
Psychological factors often maintain sleep problems whatever their original cause. Children may have got into the habit of waking up at night and have been encouraged by the parental response (see below). They may be anxious not to be separted from a parent, fearing that the parent will be injured, leave, or die during the night. This anxiety will be more common among children who have witnessed marital violence, undergone a long separation, or experienced life threatening illness or death of a loved one. Those children who wake at night to find that they are being sexually abused will have particular fears of falling asleep.

Psychiatric disorders-Occasionally among older chidren and adolescents sleep disturbance may be related to a specific psychiatric disorder.

Generally poor sleep as well as early morning waking may be the result of a depressive illness. Misuse of drugs such as amphetamines will lead to overarousal and distorted sleep patterns. The checking associated with a severe obsessive-compulsive disorder may last until the early hours of the morning.

Parents' response - This is of prime importance. Firstly, they may fail to make going to bed an agreeable experience. The importance of a routine should not be overlooked-for example, a fixed time, regular sequence of events, calming activities such as a bath and a story, and the security of having a light on at night. Secondly, giving in for a few minutes after a prolonged battle about going to bed inadvertently rewards the child, and is enough to reinforce the behaviour pattern for long periods. Thirdly, parents may unwittingly encourage sleep problems by rewarding wakefulness and calling out with long chats, stories, cuddles, and affection. Sometimes, however, there is an underlying problem in the relationship, with one parent emotionally overinvolved with the child-for example, they may want the child in bed with them to relieve their own loneliness or anxiety, finding separation unbearable as the child is especially precious to them. In this case there will be a general failure to promote the child's independence and separate identity. An absent or distant relationship with the partner may exacerbate this.

\section{History and examination}

\begin{tabular}{|c|c|}
\hline \multicolumn{2}{|c|}{ Example of a sleep diary } \\
\hline Day & Monday \\
\hline Time woke in & 0600 \\
\hline Sleeps during the & \\
\hline day & $1110-1150,1430-1600$ \\
\hline Bedtime & 1900 \\
\hline $\begin{array}{l}\text { What happened at } \\
\text { bedtime? }\end{array}$ & $\begin{array}{l}\text { Cried for } 10 \text { minutes } \\
\text { and came into } \\
\text { lounge, sat quietly } \\
\text { and fell asleep at } \\
\text { 2045. Carried to bed }\end{array}$ \\
\hline $\begin{array}{l}\text { Contacts during the } \\
\text { night }\end{array}$ & $\begin{array}{l}0130 \text { Climbed into our } \\
\text { bed and slept } \\
\text { quietly until } \\
\text { morning }\end{array}$ \\
\hline
\end{tabular}

\section{Management}

\section{Devising a behaviour modification programme}
- Identify the problem
- Specify desirable and undesirable behaviours
- Plan responses to unwanted behaviour
- Plan rewards for desired behaviour
- Plan details of implementation
- Implement
- Review and, if necessary, modify

A carefully and sensitively taken history will identify relevant factors. A sleep diary is a particularly useful way of obtaining information about the problem behaviour and its maintenance. Details of the child's sleeping and waking, and the parents' responses, are recorded for one or two weeks. The child's general development and emotional wellbeing should be evaluated, including social and educational functioning.

Assessment of the family should include their current predicament and concerns, including emotional issues, financial difficulties, and unemployment. Depression or anxiety in the parents, or discord in their relationship, are relevant because they are likely to be picked up by the child whose sleep disturbance may in turn perpetuate them. Separations, whether because of death, illness, divorce, or other reasons, are likely to increase the child's anxiety. Physical examination should include inspection of the throat for tonsillar enlargement and of the chest for signs of asthma, and measurement of height and weight.
A transient, isolated, mild problem may resolve with reassurance. Otherwise behaviour modification may be helpful.

The introduction of a regular bedtime routine may signal a new regimen, and a new determination on the part of the parents, which after some initial testing out by the child is often accepted surprisingly readily. The parents should be helped to stop rewarding the child with attention or drinks for repeatedly leaving the bedroom or calling out. With encouragement most parents find that they can give enough comfort to reassure the child and themselves, but not so much as to encourage repetition of the problem behaviour. Greater rewards in the form of praise and treats (not late nights) can be given when the child sleeps through the night, or at least does not call out. A reward system can be formalised by using a "star chart" on which 


\section{Behaviour modification}

According to the theory of "operant conditioning," a response to a situation changes according to its consequences for the person concerned.

Example of how operant conditioning can maintain sleep problems

Take the situation of the child who is put to bed, and 10 minutes later gets up and joins the parents. The likelihood of the child's behaviour being repeated will depend on what happens next. A consequence which is experienced by the child as desirable (such as increased parental attention) will encourage repetition. A consequence which is not experienced as desirable (such as being promptly returned to the bedroom) will not have this effect. The parents may therefore modify the child's behaviour by avoiding rewarding behaviour that they find undesirable, and instead rewarding that which is wanted. In this case the parents should not only avoid rewards for getting up at night, but they should also institute rewards for staying in bed until morning. Inevitably, this is an extreme oversimplification of a much more complicated picture. It is, however, one which often permits effective action. stars are earned for specified behaviour such as going to bed on time, staying in bed, and not calling out. Children should not be required to do things that are beyond their control, such as going to sleep at a particular time.

Studies have shown that these programmes are extremely effective if implemented fully and conscientiously, but many parents find them difficult to put into practice. Time should therefore be spent in planning, discussing possible difficulties, and devising strategies to overcome them. Some may find it easier to change their own and the child's behaviour in small steps, progressively decreasing the time before a child who gets up is put back to bed, or the number of stories read, and so on. The initial "testing out" period is particularly strenuous for parents. They need to understand that the child is trying to re-establish the old routine, so if they waver it will increase the child's attempts to return to the old pattern.

If behavioural management proves unsuccessful, or the sleep disorder is part of a wider problem, an alternative approach may be indicated. This is likely to require the participation of child mental health services and may include psychological assessment and treatment of one form or another with the child and perhaps the parents as well. Drugs to induce sleep are likely to be of only temporary benefit and should seldom be used in children

Tony Jaffa is consultant in child and adolescent psychiatry, St George's Hospital, London; Stephen Scott is clinical lecturer in child and adolescent psychiatry, Institute of Psychiatry, London; Jean Harris Hendriks is consultant child psychiatrist, Dunstable; and Colin M Shapiro is professor of psychiatry, University of Toronto, Ontario, Canada.

The ABC of Sleep Disorders has been edited by Professor Colin M Shapiro.
Rupture of the diaphragm caused by blunt trauma is an uncommon injury in children; the condition may present late, so a high degree of awareness is essential for prompt diagnosis

\section{Children's Hospital,} Dublin 1, Ireland George Ninan, senior surgical registrar Prem Puri, consultant paediatric surgeon

\section{Correspondence to:} Mr P Puri, Children's Research Centre, Our Lady's Hospital for Sick Children, Crumlin, Dublin 12, Ireland.

\title{
Lesson of the Week
}

\section{Late presentation of traumatic rupture of the diaphragm in a child}

\author{
George Ninan, Prem Puri
}

Rupture of the diaphragm due to blunt trauma is uncommon. ${ }^{1}$ Late presentation of such an injury is well recognised in adults ${ }^{2}$ but exceptionally rare in children. ${ }^{3}$ Lack of awareness of late presentation of traumatic rupture of the diaphragm in children may result in a delay in diagnosis.

\section{Case report}

A 7 year old boy was admitted as an emergency case after falling from the side of a lorry. He was alert and neurologically normal with a Glasgow coma score of 14 on admission. He was haemodynamically stable but had a few episodes of haemoptysis, and there was macroscopic haematuria. Positive findings were decreased air entry in the right lower chest and a distended abdomen with tenderness and guarding, mainly in the suprapubic region. He was resuscitated and monitored along the lines of trauma management described by the American College of Surgeons. ${ }^{4} \mathrm{~A}$ chest $x$ ray picture showed right sided pulmonary bruising, and an abdominal film showed a fracture through each pubic ramus. Ultrasound study of the abdomen showed free fluid in the peritoneal cavity and a right perirenal collection of fluid but normal kidneys, liver, spleen, and bladder. He was managed conservatively, the fractured pelvis being treated by rest in bed. He recovered rapidly. Three days after admission ultrasonic appearances in the abdomen were normal. He continued to improve and was discharged home 11 days after admission. At discharge, when he was fully well, he was eating normally and had no clinical abnormality in chest or abdomen. A radiograph of the chest was reported on as normal (figure).

Eighteen days after discharge from hospital he was readmitted as an emergency complaining of epigastric pain and vomiting for 24 hours. On examination he looked pale and dehydrated. He was in no obvious respiratory distress but the chest sounded dull on percussion and there was decreased air entry on the left. A chest $x$ ray picture showed features interpreted as consolidation of the left lower lobe with a left sided pleural effusion (figure). A provisional diagnosis of staphylococcal pneumonia was made. He was treated with intravenous fluids and antibiotics.

Eight hours after admission he suddenly deteriorated, with severe respiratory distress and increasing left chest signs. Review of the chest $x$ ray picture suggested post-traumatic diaphragmatic hernia, which was confirmed by ultrasound examination. During transfer to the intensive care unit he suffered a cardiac arrest which necessitated 20 minutes of cardiopulmonary resuscitation to establish a normal cardiac rhythm. At laparotomy, performed after a period of attempted stabilisation, the whole of the stomach and omentum was found to have herniated into the left hemithorax. The hugely distended stomach had to be decompressed before it could be returned back into the abdomen, and the defect in the left hemidiaphragm $(6 \times 5 \mathrm{~cm})$ was repaired with 2-0 silk sutures. He had an 